\title{
Fatores associados a uma pior avaliação da qualidade de vida entre familiares cuidadores de usuários de Centros de Atenção Psicossocial
}

\author{
Factors associated with worst quality of life assessment \\ among family caregivers of users from Psychosocial Care \\ Centers
}

\author{
Luciane Prado Kantorski ${ }^{1}$, Vanda Maria da Rosa Jardim¹, \\ Carlos Alberto dos Santos Treichel ${ }^{1}$, Christian Loret de Mola ${ }^{1}$, \\ Poliana Farias Alves ${ }^{1}$, Roberta Zaffalon Ferreira ${ }^{1}$, Michele Mandagará de Oliveira ${ }^{1}$
}

\begin{abstract}
Resumo
Introdução: A identificação dos fatores associados a uma pior avaliação da qualidade de vida dos familiares cuidadores em saúde mental pode constituir uma estratégia importante para o estabelecimento de ações de acompanhamento e de suporte desses sujeitos. Objetivo: Este estudo buscou identificar quais os fatores associados a uma pior avaliação da qualidade de vida entre 1.242 familiares cuidadores de usuários de 40 Centros de Atenção Psicossocial (CAPS) localizados no Sul do Brasil. Métodos: Para análise, munindo-se de um modelo teórico hierarquizado, utilizou-se a regressão logística com o cálculo das razões de odds ajustadas. Resultados: Os fatores associados a uma pior avaliação da qualidade de vida foram: escolaridade, facilidade de acesso aos CAPS, eficácia dos CAPS, apoio dos CAPS na sobrecarga, divisão das atividades do cuidado, problema de saúde, relação com a família e sentimento de sobrecarga. Conclusão: Ao considerar a gama de aspectos relacionados ao serviço que estiveram associados ao desfecho, este estudo entende que os CAPS exercem um papel importante na vida dos familiares cuidadores a eles vinculados, influenciando significativamente em sua qualidade de vida.
\end{abstract}

Palavras-chave: qualidade de vida; cuidadores; saúde mental; serviços comunitários de saúde mental.

\begin{abstract}
Introduction: The identification of the factors associated with a worse assessment of the quality of life among family caregivers in mental health can be an important strategy for establishing follow-up and support actions to the subjects. Objective: This study aims to identify the factors associated with worse assessment of quality of life among 1242 family caregivers of users from 40 Psychosocial Care Centers located in southern Brazil. Methods: We used a logistic regression analysis based in a hierarchical theoretical model to calculate the adjusted odds ratios. Results: Factors associated with worse assessment of quality of life were: education; access the Psychosocial Care Center; effectiveness of Psychosocial Care Center; Psychosocial Care Center support in cases of burden; share of care activities; health problem; family relationship and feeling of burden. Conclusion: Considering the range of issues related to service that were associated with the outcome, this study highlight the prospect that the Psychosocial Care Centers play an important role in the lives of family caregivers, significantly influencing their quality of life.

Keywords: quality of life; caregivers; mental health; community mental health services.
\end{abstract}

\footnotetext{
${ }^{1}$ Departamento de Enfermagem, Universidade Federal de Pelotas (UFPel) - Pelotas (RS), Brasil. Trabalho realizado em 40 Centros de Atenção Psicossocial localizados na Região Sul do Brasil. Endereço para correspondência: Luciane Prado Kantorski - R. Gomes Carneiro, 1 - Porto - CEP: 96010-610 - Pelotas (RS), Brasil - Email: kantorski@uol.com.br Fonte de financiamento: Pesquisa financiada pelo Ministério da Saúde por meio do Termo de Cooperação nº 186/2010 junto a Universidade Federal de Pelotas. Conflito de interesses: nada a declarar.
} 


\section{INTRODUÇÃO}

Em serviços comunitários de saúde mental que trabalham na perspectiva de reabilitação psicossocial, como os Centros de Atenção Psicossocial (CAPS), a figura do familiar é considerada essencial para o cuidado dos indivíduos que convivem com algum transtorno mental. Ressalta-se que, no contexto do cuidado domiciliar, a família é, frequentemente, a responsável por prestar os cuidados mais importantes para essas pessoas, tornando-se parceira no processo de reabilitação ${ }^{1,2}$.

No papel de cuidadores, os familiares vivenciam tarefas múltiplas e desafiadoras para as quais, muitas vezes, não estão preparados. Embora tenham sentimentos positivos em relação ao seu ente querido, por vezes podem apresentar dificuldades em lidar com suas emoções diante de uma realidade de dúvidas e de incertezas. Destaca-se ainda a realização de mudanças importantes e significativas em seus modos de vida e rotina, sendo necessário um processo de adaptação constante que repercute diretamente em seus estilos de vida ${ }^{3,4}$.

Nesse contexto, alguns estudos ${ }^{5,6}$ têm apontado implicações na qualidade de vida dos familiares, definida pela Organização Mundial de Saúde como a percepção do indivíduo sobre a sua posição na vida, no contexto da cultura e dos sistemas de valores nos quais ele vive, e em relação a seus objetivos, expectativas, padrões e preocupações ${ }^{7}$.

Contudo, em revisão de literatura, pode-se evidenciar que as investigações nessa área ainda são escassas no contexto brasileiro. Embora haja uma produção significativa quanto à qualidade de vida de cuidadores em diversos tipos de necessidades em saúde ${ }^{8}$, em especial em relação aos idosos ${ }^{9-12}$, no campo da saúde mental são rastreados poucos trabalhos. Até o momento, não foram desenvolvidos estudos, por exemplo, para a detecção dos fatores associados a uma baixa avaliação da qualidade de vida dos cuidadores familiares em saúde mental.

A identificação dos fatores associados a uma pior avaliação da qualidade de vida dos familiares cuidadores em saúde mental pode constituir uma estratégia importante para o estabelecimento de ações de acompanhamento e de suporte desses sujeitos. Nesse sentido, este estudo objetivou identificar a prevalência e os fatores associados a uma pior avaliação da qualidade de vida dos familiares de usuários de CAPS na região Sul do Brasil.

\section{METODOLOGIA}

Trata-se de um estudo transversal com familiares de usuários de CAPS do tipo I, II e III, parte integrativa de uma pesquisa de avaliação de serviços de saúde mental de base comunitária na região Sul do Brasil (CAPSUL II) realizada em 2011.

A coleta de dados ocorreu em 40 CAPS do tipo I, II e III distribuídos no Sul do Brasil, respeitando-se a proporcionalidade de serviços em cada Estado. Assim, foram incluídos na amostra
12 serviços do Estado do Paraná, 10 do Estado de Santa Catarina e 18 do Estado do Rio Grande do Sul.

Com base no cálculo de amostra, pretendeu-se aplicar o questionário a 1.600 familiares. Para prevalência, o cálculo de amostra considerou uma frequência estimada de $50 \%$, com margem de 3 pontos, e alfa (a) de 5\%, resultando na necessidade de um $\mathrm{N}=1.066$. Para associação, utilizando um poder de amostra de $90 \%$, com confiança de $95 \%$, relação de não expostos/expostos de 2:1, risco relativo de 1,3 e prevalência de $40 \%$ em não expostos, obteve-se um indicativo de amostra de $\mathrm{N}=1.038$. Logo, foram acrescidos ao maior $\mathrm{N}$ indicado $(\mathrm{N}=1.066) 50 \%$ de indivíduos, a fim de considerar perdas e controle de fator de confusão.

A seleção dos entrevistados foi realizada por meio de amostragem não probabilística. Todos os familiares que frequentaram o serviço no período de coleta (uma semana) foram abordados. Além destes, a fim de completar a amostra, por meio dos registros dos serviços foram rastreados outros familiares, aos quais foram realizadas visitas domiciliares. O percentual de perdas no universo dos familiares correspondeu a $23 \%$, relacionado a recusas e dificuldades em encontrá-los; sendo assim, a população final para este estudo foi de 1.242 familiares.

A coleta foi realizada por 40 entrevistadores treinados previamente. O controle de qualidade dos dados foi realizado na codificação dos instrumentos de coleta, na revisão realizada pelos supervisores ao receber os questionários e na replicação de $5 \%$ das entrevistas conduzidas. A entrada dos dados no banco ocorreu por meio de dupla digitação no software EPI-INFO 6.04. Diferenças entre os dados foram comparadas, avaliadas e corrigidas quando necessário.

O desfecho deste estudo foi a avaliação dos indivíduos quanto à sua qualidade de vida, com a utilização da seguinte questão: Marque na escala abaixo como o(a) Sr.(a) se sentiu a respeito da sua qualidade de vida nas últimas quatro semanas. Das respostas possíveis, havia uma escala do tipo Likert com pontuações de 0 a 10. Para realização deste trabalho, após um estudo quanto à distribuição da amostra, foi identificada a mediana e optou-se por utilizar como ponto de corte uma pontuação igual a 8. Logo, indivíduos que atribuíram à sua qualidade de vida pontuações iguais ou inferiores a 7 foram considerados como aqueles que apresentaram uma pior avaliação de sua qualidade de vida.

Para análise, foram verificados a distribuição proporcional e os intervalos de confiança de 95\% (IC95\%) para as variáveis categóricas. Na análise bruta, a prevalência de melhora percebida foi calculada para cada variável independente, e o teste do qui-quadrado para heterogeneidade foi utilizado a fim de identificar diferenças estatisticamente significativas entre os grupos $(\mathrm{p}<0,05)$.

A análise ajustada objetivou controlar possíveis fatores de confusão em relação às variáveis do mesmo nível e àquelas de 
níveis anteriores; para isso, utilizou-se a regressão logística, com o cálculo das razões de odds ajustadas. Foi usado o teste de Wald para heterogeneidade. Os modelos de regressão levaram em consideração um modelo hierárquico que incluiu as variáveis em cinco níveis. No primeiro, como determinante distal, foram incluídas as variáveis: sexo (feminino e masculino) e idade (18 a 40 anos; 41 a 50 anos; 51 a 60 anos; 61 anos ou mais). No segundo nível, foram incluídas as variáveis: estado civil (com companheiro e sem companheiro) e escolaridade (sem escolaridade; até 4 anos de estudo; entre 5 e 8 anos de estudo; entre 9 e 11 anos de estudo; 12 anos de estudo ou mais). No terceiro nível, constaram: trabalho (possui e não possui), renda (mais de 3 salários mínimos; até 3 salários mínimos; até 2 salários mínimos; até 1 salário mínimo) e gastos com o paciente (até $25 \%$ da renda familiar; de 26 a $50 \%$ da renda familiar; de 51 a $75 \%$ da renda familiar; mais de $75 \%$ da renda familiar). No quarto nível, foram incluídos: facilidade de acesso aos CAPS (fácil acesso; intermediário; difícil acesso; não frequenta o serviço), eficácia dos CAPS (ajudou a lidar melhor com o problema e não ajudou ou ajudou parcialmente), apoio dos CAPS na sobrecarga (não se sentem sobrecarregados; recebem apoio dos CAPS na sobrecarga; não recebem apoio dos CAPS na sobrecarga), grupo de familiares nos CAPS (existe e o familiar participa; não existe; existe, mas o familiar não participa; não souberam responder sobre a existência de grupos) e divisão das atividades do cuidado (divide o cuidado e cuida sozinho). No quinto nível, como determinante proximal, foram incluídos: problema de saúde (não possui e possui), relação com a família (satisfeito; mais ou menos satisfeito; insatisfeito) e sentimento de sobrecarga (não sobrecarregado; pouco sobrecarregado; mais ou menos sobrecarregado; muito sobrecarregado).

O estudo foi submetido e aprovado, sob ofício de no 176/2011, pelo Comitê de Ética da Faculdade de Enfermagem da Universidade Federal de Pelotas, seguindo as Normas e Diretrizes Regulamentadoras da Pesquisa Envolvendo Seres Humanos - Resolução CNS no 466/2012. Os princípios éticos foram assegurados por meio de: consentimento livre e esclarecido, garantia do direito de não participação na pesquisa e anonimato.

\section{RESULTADOS}

A amostra deste estudo foi de 1.242 sujeitos. Sua composição compreendeu majoritariamente indivíduos do sexo feminino (67,8\%), com idade entre 46 e 65 anos (48,1\%), que possuíam companheiro $(60,9 \%)$, eram os únicos cuidadores do usuário $(65,4 \%)$, possuíam escolaridade igual ou inferior a 8 anos de estudo (65,3\%), sem trabalho remunerado $(60,6 \%)$ e com renda de até dois salários mínimos (34,2\%).

A proporção de familiares que realizaram uma pior avaliação de sua qualidade de vida foi de $41,32 \%$ dos entrevistados. $\mathrm{Na}$ análise bruta, estiveram associadas ao desfecho as variáveis: idade ( $\mathrm{p}<0,001)$, escolaridade $(\mathrm{p}=0,013)$, facilidade de acesso aos CAPS ( $\mathrm{p}=0,021)$, eficácia dos CAPS $(\mathrm{p}=0,003)$, apoio dos CAPS na sobrecarga $(\mathrm{p}<0,001)$, problema de saúde $(\mathrm{p} \leq 0,001)$, relação com a família $(\mathrm{p} \leq 0,001)$ e sentimento de sobrecarga $(\mathrm{p}<0,001)$

$\mathrm{Na}$ análise ajustada, nenhuma das variáveis citadas deixou de estar associada ao desfecho. No entanto, ganhou força de associação a variável divisão das atividades do cuidado $(\mathrm{p}=0,044)$.

A Tabela 1 dispõe a prevalência do desfecho estudado para cada estrato das variáveis, bem como os valores das razões de odds nas análises bruta e ajustada e seus respectivos p-valores.

Para a variável idade, o estrato com pior avaliação da qualidade de vida foi o dos indivíduos entre 51 e 60 anos, com chances $53 \%$ maiores quando comparados àqueles entre 18 e 40 anos. No mesmo sentido, chances $36 \%$ maiores também foram encontradas para indivíduos entre 41 e 50 anos.

Indivíduos com escolaridade entre 9 a 11 anos de estudo e com escolaridade entre 5 a 8 anos de estudo apresentaram chances maiores de apresentar o desfecho ( 20 e $48 \%$, respectivamente). Contudo, indivíduos com até 4 anos de estudo apresentaram chances praticamente iguais de avaliar pior sua qualidade de vida ao grupo com maior escolaridade. Já os sem escolaridade apresentaram chances $35 \%$ menores de avaliar pior sua qualidade de vida.

Comparados aos indivíduos que referiram facilidade de acesso aos CAPS, aqueles que consideraram o acesso ao serviço intermediário ou difícil apresentaram, respectivamente, chances 53 e 55\% maiores de realizar uma pior avaliação de sua qualidade de vida. No sentido inverso, os que referiram não acessar os CAPS apresentaram chances $17 \%$ menores.

Indivíduos que referiram entender que o CAPS não ajudou ou ajudou parcialmente o usuário em acompanhamento demonstraram chances $90 \%$ maiores de avaliar pior sua qualidade de vida. Quanto a receber apoio dos CAPS quando sobrecarregados, os indivíduos apresentaram chances 2,09 vezes maiores de pior avaliação da qualidade de vida quando comparados aos não sobrecarregados. Chances ainda maiores foram encontradas para aqueles que, embora sobrecarregados, referiram não receber apoio do serviço. Para eles, as chances de apresentar uma pior avaliação da qualidade de vida foram 2,56 vezes maiores.

Embora não houvesse apresentado associação na análise bruta, quando inserida no modelo, a variável "divisão das atividades do cuidado" passou a apresentar força de associação. Contrariando a perspectiva inicial de que dividir o cuidado seria preditor de melhor qualidade de vida, indivíduos que não dividiram o cuidado apresentaram chances $30 \%$ menores de avaliar pior sua qualidade de vida.

Aqueles que referiram possuir algum problema de saúde apresentaram chances $51 \%$ maiores de avaliar pior sua qualidade 
Tabela 1. Prevalência e razões de chances brutas e ajustadas da pior avaliação da qualidade de vida, em relação às características avaliadas, dos familiares de usuários de CAPS no Sul do Brasil.

\begin{tabular}{|c|c|c|c|c|c|c|}
\hline & $\mathbf{N}$ & $\%$ & OR bruto/IC (95\%) & P-valor & OR ajustado/IC (95\%) & P-valor \\
\hline \multicolumn{7}{|l|}{ Sexo } \\
\hline Masculino & 398 & 36,9 & 1 & \multirow[t]{2}{*}{0,086} & 1 & \multirow[t]{2}{*}{0,260} \\
\hline Feminino & 837 & 41,9 & $1,23(0,97-1,56)$ & & $1,15(0,89-1,48)$ & \\
\hline \multicolumn{7}{|l|}{ Idade } \\
\hline 51 a 60 & 321 & 43,9 & 1 & \multirow[t]{4}{*}{0,000} & 1 & \multirow[t]{4}{*}{0,000} \\
\hline 18 a 40 & 302 & 37,7 & $0,77(0,54-1,09)$ & & $0,77(0,54-1,09)$ & \\
\hline 41 a 50 & 254 & 48,8 & $1,21(0,87-1,70)$ & & $1,21(0,86-1,69)$ & \\
\hline 61 ou mais & 342 & 34,2 & $0,66(0,48-0,90)$ & & $0,67(0,49-0,92)$ & \\
\hline \multicolumn{7}{|l|}{ Escolaridade } \\
\hline Até 4 anos de estudo & 547 & 39,8 & 1 & \multirow[t]{5}{*}{0,013} & 1 & \multirow[t]{5}{*}{0,024} \\
\hline Sem escolaridade & 131 & 29,7 & $0,63(0,46-0,88)$ & & $0,65(0,45-0,93)$ & \\
\hline Entre 5 e 8 anos de estudo & 162 & 49,3 & $1,47(1,00-2,14)$ & & $1,48(1,00-2,20)$ & \\
\hline Entre 9 e 11 anos de estudo & 275 & 42,5 & $1,11(0,81-1,52)$ & & $1,20(0,84-1,72)$ & \\
\hline 12 anos de estudo ou mais & 111 & 39,6 & $0,99(0,67-1,44)$ & & $1,00(0,67-1,49)$ & \\
\hline \multicolumn{7}{|l|}{ Estado civil } \\
\hline Com companheiro & 750 & 41,1 & 1 & \multirow[t]{2}{*}{0,416} & 1 & \multirow[t]{2}{*}{0,988} \\
\hline Sem companheiro & 482 & 39,2 & $0,92(0,76-1,12)$ & & $1,00(0,83-1,19)$ & \\
\hline \multicolumn{7}{|l|}{ Trabalho } \\
\hline Possui trabalho remunerado & 488 & 42,4 & 1 & \multirow[t]{2}{*}{0,120} & 1 & \multirow[t]{2}{*}{0,461} \\
\hline Não possui trabalho remunerado & 747 & 38,9 & $0,86(0,72-1,03)$ & & $0,90(0,69-1,17)$ & \\
\hline \multicolumn{7}{|l|}{ Renda } \\
\hline Mais de 3 salários mínimos & 274 & 38,7 & 1 & \multirow[t]{4}{*}{0,747} & 1 & \multirow[t]{4}{*}{0,621} \\
\hline Até 3 salários mínimos & 276 & 42,4 & $1,16(0,84-1,61)$ & & $1,30(0,87-1,94)$ & \\
\hline Até 2 salários mínimos & 390 & 38,9 & $1,01(0,76-1,33)$ & & $1,19(0,81-1,75)$ & \\
\hline Até 1 salário mínimo & 196 & 41,3 & $1,11(0,78-1,59)$ & & $1,22(0,8-1,87)$ & \\
\hline \multicolumn{7}{|l|}{ Gastos com o paciente } \\
\hline Até $25 \%$ da renda familiar & 467 & 39,2 & 1 & \multirow[t]{4}{*}{0,301} & 1 & 0,432 \\
\hline De 26 a $50 \%$ da renda familiar & 283 & 37,8 & $0,94(0,72-1,23)$ & & $0,95(0,70-1,28)$ & \\
\hline De 51 a $75 \%$ da renda familiar & 138 & 47,8 & $1,42(0,94-2,15)$ & & $1,40(0,89-2,22)$ & \\
\hline Mais de $75 \%$ da renda familiar & 134 & 43,2 & $1,18(0,84-1,65)$ & & $1,09(0,75-1,58)$ & \\
\hline Facilidade de acesso aos CAPS & & & & & & \\
\hline Fácil acesso & 777 & 38,4 & 1 & 0,021 & 1 & 0,050 \\
\hline Intermediário & 144 & 47,2 & $1,43(1,05-1,94)$ & & $1,53(0,99-2,36)$ & \\
\hline Difícil acesso & 171 & 48,5 & $1,50(1,02-2,21)$ & & $1,55(0,95-2,51)$ & \\
\hline Não realiza acesso & 141 & 34,0 & $0,82(0,56-1,19)$ & & $0,83(0,54-1,29)$ & \\
\hline Eficácia dos CAPS & & & & & & \\
\hline Ajudou a lidar melhor com o problema & 1110 & 39,1 & 1 & 0,003 & 1 & 0,001 \\
\hline Não ajudou ou ajudou parcialmente & 116 & 50,8 & $1,61(1,17-2,20)$ & & $1,90(1,31-2,74)$ & \\
\hline Apoio dos CAPS na sobrecarga & & & & & & \\
\hline Não se sentem sobrecarregados & 577 & 30,8 & 1 & 0,000 & 1 & 0,000 \\
\hline $\begin{array}{l}\text { Recebem apoio dos CAPS na } \\
\text { sobrecarga }\end{array}$ & 335 & 47,1 & $2,00(1,58-2,53)$ & & $2,09(1,57-2,78)$ & \\
\hline $\begin{array}{l}\text { Não recebem apoio dos CAPS na } \\
\text { sobrecarga }\end{array}$ & 290 & 48,9 & $2,15(1,62-2,83)$ & & $2,56(1,86-3,42)$ & \\
\hline Grupo de familiares nos CAPS & & & & & & \\
\hline Existe e o familiar participa & 354 & 42,1 & 1 & 0,650 & 1 & 0,081 \\
\hline Não existe & 272 & 37,8 & $0,83(0,58-1,19)$ & & $0,64(0,41-1,01)$ & \\
\hline Existe, mas o familiar não participa & 255 & 43,5 & $1,06(0,72-1,54)$ & & $1,02(0,67-1,54)$ & \\
\hline $\begin{array}{l}\text { Não souberam responder sobre a } \\
\text { existência de grupos }\end{array}$ & 352 & 38,3 & $0,85(0,57-1,26)$ & & $0,65(0,44-0,97)$ & \\
\hline Divisão das atividades do cuidado & & & & & & \\
\hline Divide o cuidado & 428 & 41,3 & 1 & 0,687 & 1 & 0,044 \\
\hline Cuida sozinho & 805 & 39,8 & $0,94(0,69-1,26)$ & & $0,70(0,49-0,99)$ & \\
\hline
\end{tabular}

OR: Odds Ratio; CAPS: Centros de Atenção Psicossocial 
Tabela 1. Continuação...

\begin{tabular}{|c|c|c|c|c|c|c|}
\hline & $\mathbf{N}$ & $\%$ & OR bruto/IC (95\%) & P-valor & OR ajustado/IC (95\%) & P-valor \\
\hline \multicolumn{7}{|l|}{ Problema de saúde } \\
\hline Não possui & 572 & 34,4 & 1 & \multirow[t]{2}{*}{0,000} & 1 & \multirow[t]{2}{*}{0,007} \\
\hline Possui & 663 & 45,4 & $1,58(1,26-1,98)$ & & $1,51(1,12-2,04)$ & \\
\hline \multicolumn{7}{|l|}{ Relação com a família } \\
\hline Satisfeito & 974 & 35,7 & 1 & \multirow[t]{3}{*}{0,000} & 1 & \multirow[t]{3}{*}{0,003} \\
\hline Mais ou menos satisfeito & 148 & 51,3 & $1,89(1,22-2,94)$ & & $1,71(1,07-2,73)$ & \\
\hline Insatisfeito & 111 & 65,7 & $3,45(2,22-5,36)$ & & $2,16(1,31-3,58)$ & \\
\hline \multicolumn{7}{|l|}{ Sentimento de sobrecarga } \\
\hline Não sobrecarregado & 577 & 30,8 & 1 & \multirow[t]{4}{*}{0,000} & 1 & \multirow[t]{4}{*}{0,000} \\
\hline Pouco sobrecarregado & 103 & 39,8 & $1,48(1,02-2,14)$ & & $1,49(0,84-2,64)$ & \\
\hline Mais ou menos sobrecarregado & 224 & 46,8 & $1,97(1,51-2,58)$ & & $2,59(1,76-3,79)$ & \\
\hline Muito sobrecarregado & 327 & 52,3 & $2,45(1,82-3,30)$ & & $2,39(1,57-3,64)$ & \\
\hline
\end{tabular}

OR: Odds Ratio; CAPS: Centros de Atenção Psicossocial

de vida quando comparados aos que não mencionaram nenhum problema de saúde.

Quanto ao relacionamento intrafamiliar, indivíduos insatisfeitos com suas relações familiares apresentaram chances 2,16 vezes maiores de pior avaliação da qualidade de vida; no mesmo sentido, os mais ou menos satisfeitos tiveram chances $71 \%$ maiores.

Para sentimento de sobrecarga, quanto maior o grau de sobrecarga, maiores as chances de apresentar o desfecho. Enquanto para os pouco sobrecarregados as chances foram 49\% maiores, para os mais ou menos sobrecarregados e para os muito sobrecarregados as chances foram, respectivamente, 2,39 e 2,59 vezes maiores.

\section{DISCUSSÃO}

Diante das mudanças ocorridas nos processos de cuidado a partir da reforma psiquiátrica, na qual os familiares foram incluídos no processo terapêutico como cuidadores, considerar as repercussões na qualidade de vida dos familiares que cuidam de indivíduos convivendo com transtorno mental mostra-se necessário, visto que estudos, como os de Litzelman et al. ${ }^{5} \mathrm{e}$ de Nguyen et al. ${ }^{6}$, indicam que cuidadores primários de indivíduos com doenças crônicas apresentam pior avaliação da qualidade de vida em relação à população geral.

Uma estratégia importante diante dessa perspectiva seja, talvez, a identificação de fatores associados às repercussões negativas na vida dos familiares cuidadores, a fim de planejar estratégias de intervenções eficazes que considerem os possíveis marcadores dessas situações.

No que diz respeito à idade, os resultados analisados sugerem que há uma pior avaliação da qualidade de vida entre os estratos intermediários da amostra no que se refere à idade, com melhores perspectivas presentes igualmente entre os indivíduos mais jovens e os mais idosos.
A concentração de piores perspectivas quanto à avaliação da qualidade de vida, em sua fase intermediária, dos sujeitos pode ser atribuída à perspectiva de que essa é uma fase marcante para vários processos de vida que podem ser afetados pela necessidade de cuidado de um familiar.

Baseados em resultados de estudos prévios ${ }^{13}$, maior nível de escolaridade foi utilizado como estrato de comparação. Em seu estudo conduzido com cuidadores de indivíduos com transtorno mental na cidade de Hong Kong, Wong et al. ${ }^{13}$ encontraram melhores resultados quanto à qualidade de vida entre os cuidadores com maior nível de educação. Contudo, embora essa perspectiva se confirme ao comparar indivíduos que estudaram entre 9 a 11 anos e 5 a 8 anos àqueles com 12 anos de estudo ou mais, nesta pesquisa indivíduos que estudaram até 4 anos apresentaram chances praticamente iguais aos indivíduos com 12 anos de estudo ou mais de avaliar pior sua qualidade de vida. Contrariando ainda as perspectivas iniciais, indivíduos sem escolaridade apresentaram 35\% menos chances de avaliar pior sua qualidade de vida comparados aos indivíduos com maior nível de escolaridade.

Embora contrarie as expectativas, esse quadro pode estar relacionado às perspectivas de vida dos indivíduos, já que elas podem influenciar diretamente a criticidade com que os sujeitos avaliam sua qualidade de vida. Ressalta-se que, assim como narra Martins $^{14}$, a autoavaliação da qualidade de vida representa uma avaliação das condições dos diferentes componentes ambientais baseada em juízos de valor intersubjetivos aplicados a medições ou estimativas das condições. Nesse sentido, levando em conta que a menor escolaridade está historicamente associada a piores desfechos situacionais, esse fator pode contribuir para uma avaliação menos crítica em relação à qualidade de vida entre os indivíduos com menor escolaridade.

Facilidade de acesso aos CAPS esteve associada ao desfecho; dessa forma, entende-se que a facilidade de acesso ao serviço contribui significativamente para os resultados dos serviços 
comunitários de saúde mental prestados aos familiares cuidadores dos usuários.

Em seu estudo quanto à acessibilidade e à resolubilidade da assistência em saúde mental, Quinderé et al. ${ }^{15}$ indicaram que esses dois itens correspondem a uma relação intrínseca entre a oferta de serviços, a capacidade de utilização da população e o impacto nos resultados para a população assistida. Dessa forma, assim como no estudo de Quinderé et al. ${ }^{15}$, os resultados desta pesquisa apontam a necessidade de considerar a facilidade de acesso, em especial do ponto de vista geográfico, no planejamento da distribuição e alocação de novos serviços comunitários de saúde mental.

É importante salientar ainda a existência de um grupo de familiares que referiu não acessar o serviço. Para eles, as chances de pior avaliação da qualidade de vida foram $17 \%$ menores. Embora os motivos para a não realização do acesso não tenham sido investigados, esse quadro pode estar relacionado à inexistência de vínculo com o serviço, possivelmente pela menor proximidade com o usuário ou menor gravidade do caso clínico do sujeito.

Ao identificar associação entre pior avaliação da qualidade de vida e aspectos referentes ao serviço, como eficácia e apoio na sobrecarga, este estudo tem a perspectiva de que os serviços exercem um papel importante na vida dos familiares cuidadores a eles vinculados.

Essa perspectiva se traduz, por exemplo, à medida que indivíduos que referiram que o CAPS não ajudou ou ajudou parcialmente o usuário em acompanhamento demonstraram chances $90 \%$ maiores de avaliar pior sua qualidade de vida. Resultados semelhantes já haviam sido relatados por Wong et al. ${ }^{13}$, que, em seu estudo, encontraram associação entre pior avaliação da qualidade de vida entre cuidadores familiares de pessoas que têm transtornos mentais com menor satisfação em relação aos serviços comunitários de saúde mental.

A ideia de que os serviços exercem um papel importante na vida dos familiares cuidadores a eles vinculados é apontada ainda na perspectiva de que indivíduos que não recebiam apoio do serviço quando sobrecarregados apresentavam chances consideravelmente maiores de avaliar pior a qualidade de vida do que aqueles que recebiam apoio. Somados aos achados de Hasan et al. ${ }^{16}$, esses resultados contribuem para a formação de uma base de evidência quanto à contribuição dos serviços comunitários de saúde mental para a qualidade de vida dos familiares cuidadores de seus usuários.

Nesse sentido, é necessário que os serviços estejam atentos aos impactos que produzem na vida dos familiares cuidadores. É necessário levar em conta que, na perspectiva de atenção psicossocial, a qual baliza os serviços comunitários de saúde mental, não se espera que as famílias apenas convivam e cuidem do usuário, mas que sejam compreendidas em suas dificuldades para lidar com esse fato ${ }^{17}$.
Baseando-se na perspectiva de que dividir as atividades do cuidado estivesse associada a melhores resultados na vida dos familiares cuidadores, o grupo de indivíduos que dividia o cuidado com outras pessoas foi adotado como grupo de comparação. Quadros et al. ${ }^{18}$, por exemplo, encontraram chances mais baixas de rastreio de transtornos psiquiátricos menores entre os familiares que contavam com a ajuda de outras pessoas no cuidado ao indivíduo com transtorno mental. Contudo, contrariando as expectativas, neste estudo, indivíduos que cuidavam sozinhos do usuário em acompanhamento apresentaram chances $30 \%$ menores de avaliar pior sua qualidade de vida.

Esse resultado pode estar permeado por fatores que não foram suficientemente investigados, como a posição que o usuário do serviço ocupa na estrutura familiar. Santin e Klafke ${ }^{19}$, por exemplo, citam que, quando o filho é o portador de transtorno mental, a família é afetada em uma dimensão diferente do que quando o pai e/ou a mãe adoecem. Segundo as autoras, isso ocorre pela suposta relação de dependência que o filho tem com os pais, podendo até exercer uma função positiva de unir o casal, ou, ao contrário, de agravar as tensões. Logo, a divisão do cuidado pode assumir tanto um aspecto positivo quanto negativo no contexto familiar.

Outro ponto a ser considerado diz respeito ao grau de comprometimento dos indivíduos que são cuidados por esses familiares. Ressalta-se que indivíduos com o mesmo diagnóstico podem apresentar características muito particulares quanto a seu quadro clínico, que tem como balizador o estágio de evolução do diagnóstico. Nesse sentido, sugere-se que o grau de comprometimento de cada indivíduo possa permear dois aspectos capazes de influenciar diretamente os resultados encontrados por este estudo: a necessidade ou não de mais de um cuidador e a intensidade com que a vida dos familiares é afetada pela necessidade de cuidado.

Dessa forma, indica-se que a falta de informações quanto ao indivíduo cuidado pelos familiares estudados constitui uma limitação desta pesquisa. Ressalta-se que estudos anteriores acerca da qualidade de vida em cuidadores informais apontam que, entre os fatores que foram identificados como preditores independentes consistentes de melhores condições de qualidade de vida, estão algumas características do usuário, como diagnóstico e classificação de dependência ${ }^{20}$.

Possuir problemas de saúde esteve associado a uma pior avaliação da qualidade de vida entre os cuidadores estudados. Entre os indivíduos que relataram algum problema de saúde, as chances de avaliar pior sua qualidade de vida foram $51 \%$ maiores. Resultados semelhantes foram encontrados no contexto brasileiro entre cuidadores de idosos funcionalmente prejudicados 9 .

No campo da saúde mental, possuir problemas de saúde vem sendo evidenciado como um importante marcador de repercussões negativas na vida de cuidadores de pessoas com 
transtorno mental. Em seu estudo, Treichel et al. ${ }^{21}$ apontaram que aspectos, como a manifestação de transtornos psiquiátricos menores e a sobrecarga em cuidadores familiares em saúde mental, estão associados à presença de problemas de saúde.

Nesse sentido, pode-se inferir que a associação entre problemas de saúde e pior avaliação da qualidade de vida encontrada neste estudo pode ser resultado de um processo ainda mais complexo nas relações de cuidado no contexto familiar. Alguns autores $^{9}$ têm sinalizado que cuidadores familiares, em geral, estão expostos a uma série de fatores que pode contribuir para deterioração de seu estado de saúde, como as cargas emocional e física implicadas nas atividades do cuidado.

Outro aspecto muito complexo que esteve associado a uma pior qualidade de vida neste estudo faz alusão à insatisfação com as relações familiares por parte dos cuidadores. Além de ir ao encontro de resultados prévios encontrados na literatura, como o estudo de Rodríguez-Sánchez et al. ${ }^{22}$, esse achado chama atenção para uma interface importante do cuidado no contexto familiar: a forma como a estrutura familiar pode ser afetada pelas relações de cuidado. Nesse sentido, os resultados deste estudo apontam para a necessidade de que essa variável seja levada em conta na abordagem dos serviços tanto para o monitoramento das repercussões do cuidado na vida dessas pessoas quanto para o estabelecimento do plano de cuidados do usuário e seus familiares.

Sentimento de sobrecarga esteve associado ao desfecho: quanto maior o nível de sobrecarga relatado pelos familiares acessados pelo estudo, maiores foram as chances encontradas de pior avaliação da qualidade de vida.

Ressalta-se que a sobrecarga vem sendo repetidamente citada por trabalhos no campo da saúde mental como um disparador das repercussões negativas na vida dos cuidadores familiares ${ }^{21}$; contudo, não se trata de um aspecto fácil de ser abordado, embora recorrente nesse contexto. Alguns autores citam, por exemplo, que, para alguns cuidadores, a sobrecarga é algo inerente às atividades do cuidado e está atrelada a sua responsabilidade de cuidar de "parentes doentes"13. Dessa forma, evidencia-se que, em geral, entre as expectativas desses cuidadores, não está a ausência de sobrecarga, mas a necessidade de apoio por parte dos serviços de saúde ${ }^{23}$.

Ressalta-se que este estudo evidencia a influência do apoio dos CAPS para uma melhor avaliação da qualidade de vida por parte dos cuidadores familiares em saúde mental; no entanto, os debates contemporâneos acerca da política nacional de saúde mental têm apontado novos rumos para a problematização do atendimento às demandas e necessidades desses sujeitos. Isso porque, em um sistema de saúde complexo como o Sistema Único de Saúde (SUS), que prevê a articulação de uma rede de atenção aos indivíduos assistidos, não é viável que o atendimento de determinada população esteja condicionado a um único serviço.

Embora alguns autores ${ }^{19}$ sinalizem que falar de rede de atenção no SUS ainda é bastante complicado, faz-se necessário lançar mão de novas abordagens quanto ao acompanhamento dos indivíduos com transtornos mentais e seus respectivos cuidadores. Uma alternativa viável, por exemplo, é a maior articulação da equipe dos CAPS com as Estratégias Saúde da Família (ESFs) por meio das ações de matriciamento, possibilitando um cuidado mais próximo, frequente e compartilhado entre os diversos pontos de cuidado da rede de atenção.

\section{CONCLUSÃO}

Os fatores associados a uma pior avaliação da qualidade de vida foram: escolaridade, facilidade de acesso aos CAPS, eficácia dos CAPS, apoio dos CAPS na sobrecarga, divisão das atividades do cuidado, problema de saúde, relação com a família e sentimento de sobrecarga. Dessa forma, ao considerar a gama de aspectos relacionados ao serviço que esteve associada ao desfecho, este estudo entende que os CAPS exercem um papel importante na vida dos familiares cuidadores a eles vinculados, influenciando significativamente em sua qualidade de vida.

Contudo, levando em conta a complexidade do tema e a necessidade de avanços no tópico, este estudo sinaliza a necessidade de se lançar mão de novas abordagens quanto ao acompanhamento dos usuários dos CAPS e seus respectivos cuidadores, a fim de potencializar o apoio a essa população na rede de atenção proposta pelo SUS.

O delineamento deste estudo limita a determinação das relações causais entre as variáveis e a capacidade de generalização dos resultados. No entanto, diante da escassez de estudos na área, esses resultados podem ser utilizados para iniciar maiores discussões e subsidiar a proposição de trabalhos ainda mais detalhados e com delineamentos de maior potencial preditivo e de generalização.

\section{REFERÊNCIAS}

1. Moreno V, Alencastre MB. A trajetória da família do portador de sofrimento psíquico. Rev Esc Enferm USP. 2003;37(2):43-50. http://dx.doi.org/10.1590/ S0080-62342003000200006. PMid:14606451.

2. Waidman MAP, Elsen I. Família e necessidades... revendo estudos. Acta Sci Health Sci. 2004;26(1):147-57.
3. Sant'Ana MM, Pereira VP, Borenstein MS, Silva AL. O significado de ser familiar cuidador do portador de transtorno mental. Texto Contexto Enferm. 2011;20(1):50-8. http://dx.doi.org/10.1590/S0104-07072011000100006.

4. Oliveira RMP, Loyola CM. Família do paciente psiquiátrico: o retrato de uma ilustre desconhecida. Acta Sci Health Sci. 2004;26(1):213-22. 
5. Litzelman K, Skinner HG, Gangnon RE, Javier F No, Malecki K, Witt WP. Role of global stress in the health-related quality of life of caregivers: evidence from the survey of the health of Wisconsin. Qual Life Res. 2014;23(5):156978. http://dx.doi.org/10.1007/s11136-013-0598-z. PMid:24322907.

6. Nguyen DL, Chao D, Mab G, Morgana T. Quality of life and factors predictive of burden among primary caregivers of chronic liver disease patients. Ann Gastroenterol. 2015;28(1):124-9. PMid:25608915.

7. The WHOQOL Group. The World Health Organization quality of life assessment (WHOQOL): position paper from the World Health Organization. Soc Sci Med. 1995;41(10):1403-10. http://dx.doi.org/10.1016/02779536(95)00112-K. PMid:8560308.

8. Lima ML, Santos JFL, Sawada NO, Lima LAP. Quality of life of individuals with stroke and their caregivers in a city of Triângulo Mineiro. Rev Bras Epidemiol. 2014;17(2):453-64. http://dx.doi.org/10.1590/18094503201400020013ENG. PMid:24918416.

9. Reis LA, Santos KT, Reis LA, Gomes NP. Quality of life and associated factors for caregivers of functionally impaired elderly people. Braz J Phys Ther. 2013;17(2):146-51. http://dx.doi.org/10.1590/S1413-35552012005000078. PMid:23778765.

10. Puig M, Rodriguez N, Lluch-Canut MT, Moreno C, Roldán J, Montesó P. Quality of life and care burden among informal caregivers of elderly dependents in Catalonia. Rev Port Enferm Saude Mental. 2015;14:9-14.

11. Coura AS, Nogueira CA, Alves FP, Aragão JS, França ISX, Medeiros KKA. Quality of life of caregivers of octogenarians: a study using the WHOQOLBREF. Invest Educ Enferm. 2015;33(3):529-38. PMid:28569961.

12. Pereira LSM, Soares SM. Fatores que influenciam a qualidade de vida do cuidador familiar do idoso com demência. Ciênc saúde coletiva. 2015;20(12):3839-51.

13. Wong DFK, Lam AYK, Chan SK, Chan SF. Quality of life of caregivers with relatives suffering from mental illness in Hong Kong: roles of caregiver characteristics, caregiving burdens, and satisfaction with psychiatric services. Health Qual Life Outcomes. 2012;10:15. http://dx.doi.org/10.1186/14777525-10-15. PMid:22289443.

14. Martins CHB. Pobreza, meio ambiente e qualidade de vida: indicadores para o desenvolvimento humano sustentável. Indic Econ FEE. 2002;30(3):17188.
15. Quinderé PHD, Jorge MSB, Nogueira MSL, Costa LFA, Vasconcelos MGF. Acessibilidade e resolubilidade da assistência em saúde mental: a experiência do apoio matricial. Ciênc saúde coletiva. 2013;18(7):2157-66.

16. Hasan AA, Callaghan P, Lymn JS. Evaluation of the impact of a psychoeducational intervention for people diagnosed with schizophrenia and their primary caregivers in Jordan: a randomized controlled Trial. BMC Psychiatry. 2015;15(1):72. http://dx.doi.org/10.1186/s12888-015-0444-7. PMid:25885432.

17. Mielke FB, Kohlrausch E, Olschowsky A, Schneider JF. A inclusão da família na atenção psicossocial: uma reflexão. Rev. Eletr. Enf. 2010;12(4):761-5. http://dx.doi.org/10.5216/ree.v12i4.6812.

18. Quadros LC, Gigante DP, Kantorski LP, Jardim VMR. Minor psychiatric disorders in family caregivers of users of Psychosocial Care Centers in southern Brazil. Cad Saude Publica. 2012;28(1):95-103. http://dx.doi. org/10.1590/S0102-311X2012000100010. PMid:22267069.

19. Santin G, Klafke TE. A família e o cuidado em saúde mental. BarBaroi. 2011;34:146-60.

20. Naglie G, Hogan DB, Krahn M, Black SE, Beattie BL, Patterson C, et al Predictors of Family caregiver ratings of patient quality of life in Alzheimer's disease: cross-sectional results from the Canadian Alzheimer's disease quality of life study. Am J Geriatr Psychiatry. 2011;19(10):891-901. http:// dx.doi.org/10.1097/JGP.0b013e3182006a7f. PMid:21946805.

21. Treichel CAS, Jardim VMR, Kantorski LP, Vasem ML, Neutzling AS. Clustering of minor psychiatric disorders and burden among family caregivers of individuals with mental illness. Ciênc saúde coletiva. 2016;21(2):585-90.

22. Rodríguez-Sánchez E, Pérez-Peñaranda A, Losada-Baltar A, PérezArechaederra D, Gómez-Marcos MÁ, Patino-Alonso MC, et al. Relationships between quality of life and family function in caregiver. BMC Fam Pract. 2011;12(1):19. http://dx.doi.org/10.1186/1471-2296-12-19. PMid:21496270.

23. Eloia SC, Oliveira EN, Eloia SMC, Lomeo RC, Parente JRF. Sobrecarga do cuidador familiar de pessoas com transtorno mental: uma revisão integrativa. Saúde Debate. 2014;38(103):996-1007. http://dx.doi.org/10.5935/01031104.20140085.

Recebido em: Mar. 01, 2017 Aprovado em: Fev. 21, 2018 